\title{
Epidermal growth factor receptor pathway mutation and expression profiles in cervical squamous cell carcinoma: therapeutic implications
}

Sureewan Bumrungthai ${ }^{1}$, Kavita Munjal ${ }^{2}$, Shirish Nandekar², Kumarasen Cooper ${ }^{3}$, Tipaya Ekalaksananan ${ }^{1}$, Chamsai Pientong ${ }^{1,4^{*}}$ and Mark Francis Evans $5^{5,6^{*}}$

\begin{abstract}
Background: Cervical squamous cell carcinoma (CSCC) is a major cause of female mortality worldwide. This study has examined epidermal growth factor receptor (EGFR) pathway markers that represent actionable pharmacological targets.

Methods: HPV16 positive CSCCS ( $n=105$ patients) from Madhya Pradesh, India were screened for KRAS and PIK3CA mutations by PNA-clamp real-time PCR. Immunohistochemistry (IHC) was performed for EGFR, PIK3CA, PTEN, phospho-AKT, phospho-mTOR and phospho-44/42 MAPK (ERK1/2).

Results: KRAS mutations were detected in 0/91 (0\%) and PIK3CA mutations in 19/95 (20.0\%) informative specimens: exon 9, E542 $(n=3)$ and E545 $(n=15)$; exon 20, H1047R $(n=1)$. PIK3CA mutation detection was associated with older mean patient age [48.2 vs. 56.6 years $(P=0.007)]$ and with post-menopausal age: $5 / 45(11.1 \%)$ patients $<50$ years vs. 14/50 (28.0\%) patients $\geq 50$ years $(P=0.045 ; O R=3.11)$. EGFR expression was present in 60/101 (59.4\%) CSCCs and was associated with PIK3CA mutation detection $(P<0.05)$ but not age $(P>0.05)$. EGFR and phospho-AKT staining showed associations with tumor grade and/or lymph node status $(P<0.05)$. Significant associations were not found for the other study markers $(P>0.05)$.

Conclusion: These data show that PIK3CA mutation acquisition is related to patient age and EGFR expression. The absence of KRAS mutations supports the potential of anti-EGFR therapies for CSCC treatment. The relatively high PIK3CA mutation rates indicate that PI3K may be a therapeutic target for a significant subset of CSCC patients. Qualitatively distinct IHC staining profiles for the marker panel were noted patient to patient; however, across patients, consistent linear relationships between up- and downstream pathway markers were not observed. Evaluation of the expression status of potential cancer pathway targets may be of value in addition to molecular profiling for choosing among therapeutic options.
\end{abstract}

Keywords: Cervical cancer, HPV16, EGFR, PIK3CA, KRAS, PTEN, AKT, mTOR, MAPK, ERK1/2

\section{Background}

Cervical cancer is the fourth most common and fatal female malignancy worldwide: each year there are an

\footnotetext{
*Correspondence: chapie@kku.ac.th; mark.evans@uvm.edu

1 Department of Microbiology, Khon Kaen University, Khon Kaen 40002,

Thailand

${ }^{5}$ Department of Pathology and Laboratory Medicine, University of Vermont, Burlington 05405, VT, USA

Full list of author information is available at the end of the article
}

estimated 528,000 new cases and 266,000 deaths from the disease [1]. Around $85 \%$ of deaths occur in low or middle income less developed countries (LDCs). The highest mortality rates are found in African and South Asian countries [1, 2]. India ranks first for the overall number of cervical cancer deaths per year: 72,825 , which accounts for more than a quarter of the global burden. With respect to mortality rate (15.2/100,000 deaths), India ranks seventeenth $[1,2]$. 
Cervical cancer is a preventable disease. The introduction of national cervical cytology screening programs among more developed countries (MDCs) has greatly reduced disease incidence and mortality rates [3]. Human papillomaviruses (HPV) are well characterized as the cause of cervical cancer and supplementation of cytology screening with HPV testing has also helped improve the detection of precursor lesions and patient management. HPV vaccination programs have been established in many countries. However, LDCs such as India lack the resources and infrastructure to support comprehensive national cervical cytology screening or HPV vaccination programs. Although initiatives are in place, it will likely be many years before screening and vaccination are widely accessible to Indian women, especially in rural areas where the majority of cervical cancer related deaths occur $[4,5]$. Consequently, the disease will remain a social and economic burden in India for some time to come. Additionally, cervical cancer continues to impact a significant subset of women from MDCs; in the USA, 12,900 new cases and 4,100 deaths (2.3 per 100,000) are predicted for 2015 [6]. Therefore there is a considerable need for cervical cancer treatments that are effective worldwide.

The epidermal growth factor receptor (EGFR), a receptor tyrosine kinase (RTK), is a well characterized target for anti-cancer therapies for a variety of cancer types [7, 8]. EGFR stimulation activates a tyrosine kinase domain that controls multiple cell growth and division functions including maturation, proliferation, inhibition of apoptosis, angiogenesis and metastasis via pathways downstream of EGFR signaling. The MAPK/ERK and PI3K/ AKT/mTOR pathways are of particular clinical interest $[7,8]$. EGFR mediated activation of the mitogen-activated protein kinase/extracellular signal-regulated kinase (MAPK/ERK) signaling cascade (KRAS, BRAF, MEK and ERK1/2) mobilizes transcription factors leading to cell proliferation [9-11]. Mutations to the oncogene KRAS result in constitutively active KRAS allowing chronic downstream signaling. EGFR stimulation also activates the PI3K/AKT/mTOR pathway that results in translation factor activation protein synthesis and degradation of the tumor suppressor transcription factor FOXO3; PI3K (phosphatidylinositol-4,5-bisphosphate 3-kinase) is also activated by RAS $[9,12-14]$. Mutations to the oncogene PIK3CA (a sub unit of PI3K) may predict responsiveness to PI3K/AKT/mTOR inhibitors and to anti-EGFR therapies [12-16].

Causes of EGFR upregulated expression include receptor overexpression and activating mutations [7]; however, in cervical cancer EGFR mutations are undetectable [1419] or uncommon [20]. EGFR expression in cervical cancer may also be activated by HPV16 E5 or E6/E7 proteins
[21, 22]. Additionally, HPV16 E6 has been shown to cause receptor hyperactivation under conditions of abundant growth factor availability and to increase the internalization of phosphorylated EGFR even in the absence of growth factors; this results in prolonged receptor activation and MAPK and PI3K stimulation [22].

Pharmacologically, there are two modes of EGFR inhibition: anti-EGFR monoclonal antibodies (e.g., cetuximab, panitumumab), or specific inhibitors of the EGFR tyrosine kinase domain (e.g., erlotinib, lapatinib). Clinical efficacy of anti-EGFR therapies has been shown in clinical trials for lung, colon, pancreas and head and neck tumors [7, 11-15]. Anti-EGFR treatment efficacy is impacted by aberrations to downstream elements in the pathway [7-9, 11-15]. KRAS is the most frequently mutated member of the RAS gene family and encodes a $21 \mathrm{kDa}$ guanosine 5 -triphosphate-(GTP-) binding protein early in the MAPK signaling pathway. The most common KRAS mutations occur at codon positions 12 and 13 in exon 2 , and less frequently in codons $61,63,117,119$, and 146 . Wild type KRAS protein expression is normally regulated via EGFR and GAP proteins; however, activating KRAS mutations are insensitive to EGFR or GAP inhibition resulting in chronic signaling of cells to grow and divide: activated KRAS results in the phosphorylation and activation of ERK $1 / 2$ via the activities of B-RAF and MEK 1/2. Phosphorylated ERK $1 / 2$ activates AP-1 family transcription factors such as jun and fos, which on binding to AP-1 result in the expression of cyclins, cytokines and growth factors promoting cell proliferation [7-9, 11, $12,23]$. Novel ERK inhibitors are in development to facilitate treatment of patients with activating KRAS or BRAF mutations [23].

EGFR also stimulates PI3K expression and the PI3K/ AKT/mTOR cascade resulting in increased transcription, protein synthesis and proliferation [9, 13-15]. The phosphatidylinositol-4, 5-bisphosphate 3-kinase, catalytic subunit alpha $(P I K 3 C A)$ gene encodes the $\mathrm{p} 110 \alpha$ catalytic subunit protein of PI3K. PIK3CA mutations occur in a wide range of tumors. In some tumor types PIK3CA mutations are frequently associated with EGFR or KRAS mutations [24, 25] and with a poorer prognosis [25]. A number of drugs targeting PI3K have been developed and are under investigation for therapeutic utility [18-22]. Phosphatase and tensin homolog (PTEN) is tumor suppressor gene that negatively regulates PI3K signaling by protein phosphatase activity. Loss of expression or PTEN mutation is associated with many tumors $[15,26]$.

Active PI3K, phosphorylated through receptor tyrosine kinase or RAS GTP activity, can phosphorylate the $3^{\prime}$ position hydroxyl group of the inositol ring of phosphatidylinositol (PIP2) converting it to PIP3; the serine/threonine protein kinase AKT (Protein Kinase B) binds to PIP3 
at the plasma membrane, allowing PDK1 to activate AKT by phosphorylation at Thr308. Phosphorylated AKT is a key regulator of numerous cellular and nuclear process, including the promotion of protein synthesis via a multistep protein cascade that includes activation of mTOR (mechanistic target of rapamycin) and thence activation of the translation factor S6 K that promotes protein synthesis. Deregulators of this pathway include activating mutations in the PIK3CA gene (p110 subunit) and inactivation of the phosphatase and tensin homolog (PTEN) gene. The PIK3CA gene encodes PI3-Kinase that can phosphorylate the $3^{\prime}$ position hydroxyl group of the inositol ring of phosphatidylinositol, a key signal transducer in the PI3K-AKT pathway [12, 13]. An extensive variety of drugs have been developed targeting the PI3K/AKT/ mTOR pathway. These include inhibitors of PI3K p110 $\alpha$, AKT, or mTOR, as well as dual PI3K/mTOR inhibitors [12, 13]. A schematic of the EGFR signaling pathway is shown in Fig. 1.

The aims of our study were to examine the biologic expression and potential therapeutic significance of MAPK/ERK and PI3K/AKT/mTOR pathway mediators in Indian cervical squamous cell carcinomas (CSCCs) and to compare these data to similar studies from more developed countries. CSCCs were investigated by PNAClamp PCR for KRAS and PIK3CA mutations and by immunohistochemistry (IHC) for the expression of EGFR, pMAPK (pERK1/2), PIK3CA, PTEN, pAKT, and p-mTOR.

\section{Methods}

\section{Specimens}

The study was conducted with approvals from the Institutional Review Boards of the Sri Aurobindo Institute of Medical Sciences (SAIMS), Indore, Madhya Pradesh, India, and The University of Vermont, Burlington, Vermont, USA; patient informed consent was not required as coded archival specimens from patients already treated to the standard of clinical care were utilized. Formalin-fixed, paraffin-embedded (FFPE) CSCC specimens from 105 patients, untreated other than by surgery, were accrued for the study. All specimens had previously tested HPV16 positive [27]. Patient cohort clinical data is shown in Table 1.

\section{DNA extraction}

Five to ten $10 \mu \mathrm{m}$ FFPE tumor sections were cut into $1.5 \mathrm{~mL}$ tubes. Microtome blades were cleaned with xylene, alcohol and DNA AWAY (Thermo Scientific, Waltham, MA, USA) between specimen blocks. Tissue sections were cleared of paraffin by xylene followed by ethanol washes. DNA was extracted from dehydrated tissues using proteinase $\mathrm{K}$ combined with column purification (DNeasy Blood and Tissue kit, Qiagen, Valencia, CA, USA). DNA extracts were quantified and assessed for purity by NanoDrop 2000 spectrophometry (NanoDrop Technlogies, Wilmington, DE, USA). DNA amplification quality was confirmed for all samples by PCR for a 209 base pair $\beta$-globin fragment [27].

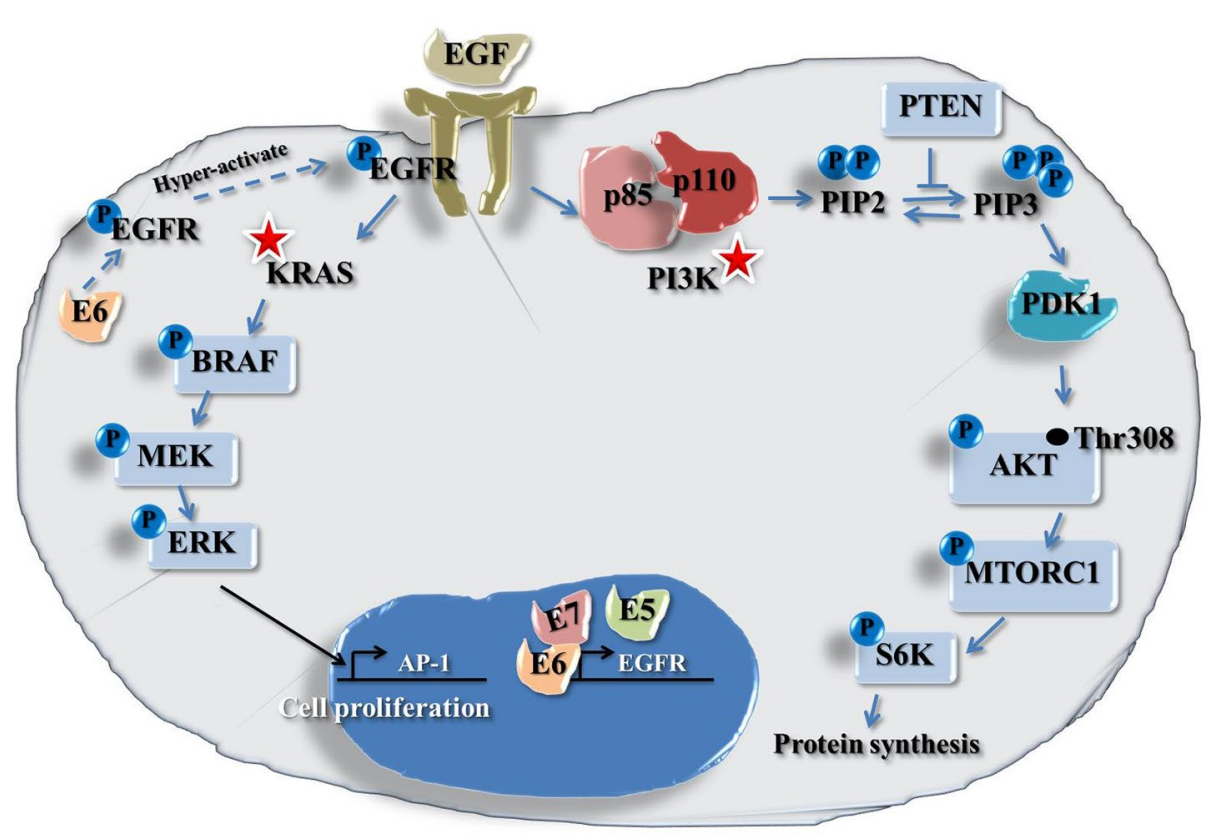

Fig. 1 Simplified representation of the EGFR MAPKJERK and PI3K/AKT/mTOR pathways (see "Background" text for step details). 
Table 1 Characteristics of the analyzed cohort

\begin{tabular}{ll}
\hline Clinical characteristics & $\mathbf{n}=\mathbf{1 0 5}$ \\
\hline Age range & $25-90$ \\
Mean age (SD) & $49.7(13.2)$ \\
FIGO stage & \\
Stage I & \\
T1/T1a1 & 2 \\
T1b1 & 65 \\
T1b2 & 27 \\
Stage II-III & 11 \\
Tumor size & \\
$\leq 2$ cm & 12 \\
$>2-5$ cm & 77 \\
$>5$ cm & 16 \\
Lymph node status & \\
Positive & 20 \\
Negative & 67 \\
No data & 18 \\
Histology & \\
Well & $17(16.2 \%)$ \\
Moderately & $74(70.5 \%)$ \\
Poorly & $14(13.3 \%)$ \\
\hline
\end{tabular}

\section{Mutational analysis of PIK3CA and KRAS by peptide nucleic acid (PNA) clamping assay}

KRAS and PIK3CA gene mutation assays were performed by quantitative real-time PCR (SYBR Green) using PNAClamp Mutation Detection Kits (PNA Bio Inc., Thousand Oaks, CA, USA): 15-20 ng of purified DNA per sample was assayed using a CFX96 Touch $^{\mathrm{TM}}$ Thermal Cycler (BioRad Inc., Hercules, CA, USA). The KRAS kit screens for seven common mutations of exon 2 (codon 12: nucleotide changes c.35G>A, c.35G>C, c.35G $>$ T, c.34G $>$ A, c.34G>C, c.34G>T; codon 13: c.38G>A). The PIK3CA kit screens for nine common mutations: exon 9 (E542 nucleotide changes: c.1624G $>$ A, c.1625A $>$ G, c.1625A>T; E545: c.1633G $>$ A, c.1634A>G, c.1635G>T) and exon 20 (H1047: c.3139C >T, c.3140A >T, c.3140A >G). Cycling conditions for all assays consisted of pre-denaturation at $94^{\circ} \mathrm{C}$ for $5 \mathrm{~min}$ followed by 40 cycles of denaturation $\left(94^{\circ} \mathrm{C}\right.$ for $\left.30 \mathrm{~s}\right)$, PNA clamping $\left(70^{\circ} \mathrm{C}\right.$ for $\left.20 \mathrm{~s}\right)$, annealing $\left(63^{\circ} \mathrm{C}\right.$ for $\left.30 \mathrm{~s}\right)$, and extension $\left(72^{\circ} \mathrm{C}\right.$ for $\left.30 \mathrm{~s}\right)$. The PNAClamp assay uses DNA primers to amplify across the locus/loci of interest in combination with an internal PNA oligo designed to anneal to the wild type form of the target. Primer extension and amplification is prevented if wild-type $K R A S$ or PIK3CA is present due to the stability of the PNA/DNA hybrid when sequences perfectly anneal, whereas a single base mismatch is highly destabilizing so permitting primer extension when a mutation is present. Mutation detection assessment was performed according to an algorithm provided with the kit and involves taking into account the cycle threshold $(\mathrm{Ct})$ values of specimen PCRs performed minus a PNA-Clamp oligo compared to the $\mathrm{Ct}$ value performed with a PNAClamp oligo. Clamping controls of known wild type DNA was performed as a negative control.

\section{Tissue microarray (TMA) preparation}

TMAs were prepared from the 105 CSCC specimens by removing $1.0 \mathrm{~mm}$ diameter cores of tumor from the FFPE blocks using a Tissue Arrayer (Beecher Instruments, Inc., Wisconsin, USA). Guided by haematoxylin and eosin (H\&E) stained slide-mounted tissues sections inkmarked for tumor areas, blocks were sampled from up to four different regions to control for heterogeneity. All 105 patient samples were contained in a total of five TMA blocks. TMA sections were H\&E stained and reviewed by a histopathologist to confirm tumor had been accurately targeted: in two instances, inadequate tumor material was sampled for IHC analyses.

\section{Immunohistochemistry (IHC)}

IHC was performed for six target antigens (Table 2): EGFR, PIK3CA, PTEN, and activated forms of AKT (i.e., phosphorylated at Thr308 by PDK1 that functions downstream of PI3K via conversion of PIP2 to PIP3), mTOR (i.e., phosphorylated at Ser2448 via PI3 kinase/AKT signaling), and MAPK (ERK1/2) (i.e., phosphorylated at Thr202 and Tyr204 by MEK1 and MEK2). The choice of IHC antibody clone was determined by literature and supplier datasheet review and by availability of clones suitable for FFPE applications. In some instances several alternative clone choices were available; it was beyond the resources available for this study to compare alternatives. As regards AKT, complete activation involves phosphorylation at Ser 473 and Ser 450 by mTORC2 [in addition to phosphorylation at Thr308 by PDK1 (Fig. 1)]. In order to be able to investigate specifically, the potential relationship of PIK3CA wild type/mutation status and PIK3CA/ PTEN expression on AKT phosphorylation expression, an antibody for AKT phosphorylated at Thr308 only was selected.

TMA sections were deparaffinized thorough xylene and rehydrated through graded ethanol followed by antigen retrieval. Slides were then treated with $3 \%$ hydrogen peroxide in $\mathrm{PBS} / 0.05 \%$ tween 20 buffer to block endogenous peroxidase activity. After this, a 15 min non-specific protein binding blocking step was performed (Protein Block, serum-free [Dako North America Inc., Carpinteria, CA, USA]). Primary antibody was then applied followed by secondary detection using a Dako EnVision+/HRP/DAB+kit and counterstaining with hematoxylin. IHC assays were 
Table 2 Details of immunohistochemical procedures

\begin{tabular}{|c|c|c|c|c|}
\hline Antigen & Antibody (clone) & Supplier & Antigen retrieval & $\begin{array}{l}\text { Antibody dilution, } \\
\text { incubation time }\end{array}$ \\
\hline EGFR & Mouse mAb (H11) & $\begin{array}{l}\text { Dako North America Inc., } \\
\text { Carpinteria, CA, USA }\end{array}$ & $\begin{array}{l}0.1 \%(\mathrm{w} / \mathrm{V}) \text { trypsin in } 40 \mathrm{mM} \\
\quad \mathrm{CaCl}_{2} / \mathrm{TBS} \mathrm{pH} 7.8,20 \mathrm{~min}, 37^{\circ} \mathrm{C}\end{array}$ & $\begin{array}{l}1: 100,30 \text { min RT then } \\
4^{\circ} \mathrm{C} \text { overnight }\end{array}$ \\
\hline PIK3CA & Rabbit pAb (LS-B5363) & $\begin{array}{l}\text { LifeSpan Biosciences, Inc., } \\
\text { Seattle, WA, USA }\end{array}$ & Citrate buffer $\mathrm{pH} 6.0^{\mathrm{a}}$ & $1: 150,30 \mathrm{~min}, \mathrm{RT}$ \\
\hline PTEN & Rabbit mAb (138G6) & $\begin{array}{l}\text { Cell Signaling Technology, } \\
\text { Danvers, MA, USA }\end{array}$ & Citrate buffer $\mathrm{pH} 6.0^{\mathrm{a}}$ & $1: 100,40 \mathrm{~min}, \mathrm{RT}$ \\
\hline Phospho-AKT (Thr308) & Mouse mAb (18F3.H11) & $\begin{array}{l}\text { Rockland Immunochemicals, } \\
\text { Inc., Gilbertsville, PA, USA }\end{array}$ & None & $1: 100,1 \mathrm{~h}, \mathrm{RT}$ \\
\hline Phospho-mTOR (Ser2448) & Rabbit mAb (49F9) & $\begin{array}{l}\text { Cell Signaling Technology, } \\
\text { Danvers, MA, USA }\end{array}$ & Citrate buffer $\mathrm{pH} 6.0^{\mathrm{a}}$ & $1: 100,40 \mathrm{~min}, \mathrm{RT}$ \\
\hline $\begin{array}{l}\text { Phospho-p44/42 MAPK } \\
\text { (Erk1/2) (Thr202/Tyr204) }\end{array}$ & Rabbit mAb (D13.14.4E) & $\begin{array}{l}\text { Cell Signaling Technology, } \\
\text { Danvers, MA, USA }\end{array}$ & Citrate buffer pH $6.0^{\mathrm{a}}$ & $1: 400,1 \mathrm{~h}, \mathrm{RT}$ \\
\hline
\end{tabular}

a Heat treatment: $105^{\circ} \mathrm{C}$ for 20 min (Decloaking Chamber, Biocare Medical, Concord, CA), then 20 min cool down.

performed according to supplier recommendations; optimization tests were also performed on breast, colon, or tonsil TMA specimens as well as the CSCC TMAs. Negative control assays were performed using IgG antibodies to Aspergillus niger glucose oxidase (Dako North America Inc.).

IHC staining patterns were reviewed (independently by MFE, SB with support from $\mathrm{KC}$ ) naive to mutation data and were scored with reference to the proportion of cells staining: $0=$ Negative; $1=$ Rare cell positive $(<1 \%) ; 2=$ Focally positive (1-25\%); $3=$ Variably positive $(25-75 \%) ; 4=$ Uniformly positive $(>75 \%)$; and in terms of staining intensity: $0=$ Negative; $1=$ weakly positive; $2=$ moderately positive; and $3=$ strong positive. These scores were summed to give an Allred score (AS) ranging from 0 to 7 [28]. Because IHC data interpretation is unstandardized, can be controversial and is dependent on the scoring approach, IHC staining was also categorized into low-level (LL) (AS <4) and high-level (HL) (AS $\geq 4$ ) staining groups [29]. This dichotomous grouping approach was taken given the limiting numbers of specimens in the study. Staining was judged among tumors relative to each other as matched specimens of normal cervical epithelium from each patient were not available for study.

\section{Statistical analysis}

Mutation and IHC data were cross-compared and compared against age, tumor grade (well, moderate, poor), stage (T1 vs. $\geq \mathrm{T} 2$ ), size (depth of invasion $\leq 2 \mathrm{~cm}$ vs. $>2 \mathrm{~cm}$ ), lymph node status (negative or positive). Patient outcome data was inaccessible for the purposes of this study. Data were assessed by Fisher's exact test, MannWhitney test, or Kruskall-Wallis test. Analyses were performed using InStat software (GraphPad Software Inc., La Jolla, CA, USA).

\section{Results}

\section{Mutation analyses}

KRAS mutations were detected in $0 / 91(0 \%)$ informative specimens (i.e., that showed a $\mathrm{Ct}$ value $\leq 30$ for the detection of KRAS in the absence of a PNA-Clamp oligo). PIK3CA mutations were detected in 19/95 (20.0\%) informative specimens. PIK3CA mutations in exon 9 were the most common: 3 (15.8\%) in E542 and 15 (78.9\%) cases in E545; 1 (5.3\%) specimen showed an exon 20 mutation at H1047R. Mutation status showed no correlation with FIGO stage, tumor size, differentiation or lymph node (LN) status. PIK3CA mutation detection was associated with older age: wild type mean age 48.2 (SD 13.7 , age range $25-90$ ) vs. mutation mean age 56.6 (SD 10.7 , age range $45-80), P=0.007$. Dividing the patients as pre-menopausal vs. post-menopausal, mutations were detected in $5 / 45(11.1 \%)$ women $<50$ years and in $14 / 50$ $(28 \%) \geq 50$ years $(\mathrm{P}=0.045$; $\mathrm{OR}=3.111)$. PIK3CA mutation data in relation to clinical characteristics are summarized in Table 3 and Additional file 1: Table S1.

\section{Immunohistochemistry}

Marker staining data in terms of 'negative, 'low', 'intermediate' and 'high' Allred scores is shown in Table 4.

\section{EGFR}

EGFR stained positive (AS $\geq 1$ ) in 60/101 (59.4\%) specimens; 38/101 (37.6\%) showed HL staining (AS $\geq 4$ ); 55/60 (91.7\%) tumors showed membranous and cytoplasmic staining, 2/60 (3.3\%) showed cytoplasmic staining and $3 / 60(5.0 \%)$ showed membranous staining. No significant correlations were detected with respect to patient age, tumor stage or size $(\mathrm{p}>0.05)$.

EGFR staining showed a significant association with PIK3CA mutation status: wild type mean AS 2.4 (SD 2.6) vs. mutation mean AS 3.8 (SD2.7), $\mathrm{P}=0.043$. HL EGFR 


\begin{tabular}{|c|c|c|c|c|}
\hline \multirow[t]{2}{*}{ Characteristics } & \multirow[t]{2}{*}{$n=95$} & \multicolumn{2}{|c|}{$\begin{array}{l}\text { PIK3CA mutant-PIK3CA wild- } \\
\text { type } \quad \text { type }\end{array}$} & \multirow[t]{2}{*}{$P$ value* } \\
\hline & & $\begin{array}{l}\text { No. of patient } \\
(n=19)\end{array}$ & $\begin{array}{l}\text { No. of patient } \\
(n=76)\end{array}$ & \\
\hline Mean age (SD) & $49.7(13.2)$ & $56.6(10.7)$ & $48.2(13.7)$ & 0.007 \\
\hline \multicolumn{5}{|l|}{ FIGO stage } \\
\hline Stage I & $84(88.4 \%)$ & $16(16.8 \%)$ & $68(71.6 \%)$ & \multirow[t]{2}{*}{0.688} \\
\hline Stage II-III & $11(11.6 \%)$ & $3(3.2 \%)$ & $8(8.4 \%)$ & \\
\hline \multicolumn{5}{|l|}{ Tumor size } \\
\hline$\leq 2 \mathrm{~cm}$ & 11 & 1 & 10 & \multirow[t]{3}{*}{0.433} \\
\hline$>2-5 \mathrm{~cm}$ & 69 & 16 & 53 & \\
\hline$>5 \mathrm{~cm}$ & 15 & 2 & 13 & \\
\hline \multicolumn{5}{|l|}{ Lymphnode } \\
\hline Positive & 17 & 5 & 12 & \multirow[t]{3}{*}{0.337} \\
\hline Negative & 61 & 11 & 50 & \\
\hline No data & 17 & & & \\
\hline \multicolumn{5}{|l|}{ Histology } \\
\hline Well & $16(16.8 \%)$ & $2(2.1 \%)$ & $14(14.7 \%)$ & \multirow[t]{3}{*}{0.547} \\
\hline Moderately & 65 (68.4\%) & $13(13.7 \%)$ & $52(54.7 \%)$ & \\
\hline Poorly & $14(14.7 \%)$ & $4(4.2 \%)$ & $10(10.5 \%)$ & \\
\hline
\end{tabular}

Italic value indicates statistically significant.

* Pearson chi square and Fisher's exact test.

Table 4 Summary of marker panel staining patterns

\begin{tabular}{|c|c|c|c|c|}
\hline Marker & Negative & Low & Intermediate & High \\
\hline $\operatorname{EGFR}(n=101)$ & $41(40.6 \%)$ & $22(21.8 \%)$ & $26(25.7 \%)$ & $12(11.9 \%)$ \\
\hline $\operatorname{PIK} 3 C A(n=101)$ & $1(1.0 \%)$ & $0(0 \%)$ & $13(12.9 \%)$ & 87 (86.1\%) \\
\hline PTEN $(n=100)$ & $69(69.0 \%)$ & $5(5.0 \%)$ & $26(26.0 \%)$ & $0(0 \%)$ \\
\hline$p-m T O R(n=101)$ & $37(36.6 \%)$ & $22(21.8 \%)$ & $41(40.6 \%)$ & $1(1.0 \%)$ \\
\hline $\begin{array}{l}\text { p-AKT cytoplasmic } \\
(n=101)\end{array}$ & $11(10.9 \%)$ & $5(5.0 \%)$ & $47(46.5 \%)$ & $38(37.6 \%)$ \\
\hline $\begin{array}{l}\text { P-AKT nuclear } \\
(n=101)\end{array}$ & $45(44.6 \%)$ & $4(4.0)$ & 31 (30.7\%) & $21(20.8 \%)$ \\
\hline $\begin{array}{l}\text { p-MAPK/Erk1/2 cyto- } \\
\text { plasmic }(n=102)\end{array}$ & $31(30$ & $14(13.7 \%)$ & $57(55.9 \%)$ & $0(0 \%)$ \\
\hline $\begin{array}{l}\text { p-MAPK/Erk1/2 } \\
\text { nuclear }(n=102)\end{array}$ & $27(26.5 \%)$ & $11(10.8 \%)$ & $64(62.7 \%)$ & $0(0 \%)$ \\
\hline
\end{tabular}

Each antibody was reviewed with respect to the proportion of cells staining: $0=$ Negative; $1=$ Rare cell positive $(<1 \%) ; 2=$ Focally positive $(1-25 \%)$; $3=$ Variably positive $(25-75 \%) ; 4=$ Uniformly positive $(>75 \%)$; and in terms of staining intensity: $0=$ Negative; $1=$ weakly positive; $2=$ moderately positive; and $3=$ strong positive. These scores were summed to give an Allred score (AS) ranging from 0 to 7 . Staining data is summarized in the above table as: Negative, $(\mathrm{AS}=0-1)$, Low $(\mathrm{AS}=2-3)$, Intermediate $(\mathrm{AS}=4-6)$, High $(\mathrm{AS}=7)$.

staining was noted in $12 / 19$ (63.2\%) PIK3CA mutated specimens and in 23/72 (31.9\%) wild type specimens $(\mathrm{P}=0.018$; OR $=3.652)$.

EGFR staining showed an association with lymph node status: mean AS for LN negative patients was 2.9 (SD $2.5)$ vs. 1.7 (SD 2.5) for lymph node positive specimens $(\mathrm{P}=0.047)$. On the basis of LL/HL categorization, $4 / 21$
(19.0\%) LN positive patients showed staining vs. 26/65 (40.0\%) LN negative patients $(\mathrm{P}=0.114$; $\mathrm{OR}=2.833)$.

AS data analysis showed an association of EGFR staining with histological grade: Well 4.3 (SD 2.5), Moderate 2.2 (SD 2.5), and Poor 3.4 (SD 2.8), $\mathrm{P}=0.029$; no significant trend was shown when the data were scored according to $\mathrm{LL} / \mathrm{HL}, \mathrm{P}=0.351$. Representative staining is shown in Fig. 2. Table 5 summarizes marker association data.

\section{Phospho-AKT}

Cytoplasmic staining (AS $\geq 1$ ) was detected in 90/101 (89.1\%) CSSCs; 85/101 (84.2\%) showed HL staining. No significant correlations were detected with respect to PIK3CA mutation status, patient age, tumor stage or size ( $\mathrm{p}>0.05)$. pAKT cytoplasmic staining showed an association with lymph node status: mean AS for LN negative patients was 4.9 (SD 2.3) vs. 5.8 (SD 2.2) for lymph node positive specimens; $\mathrm{P}=0.027$. On the basis of $\mathrm{LL} / \mathrm{HL}$ categorization, $3 / 21$ (14.3\%) LN positive patients showed staining vs. 13/65 (20.0\%) LN negative patients; $\mathrm{P}=0.75$. Cytoplasmic AS data analysis showed an association with histological grade: Well 6.6 (SD 0.5), Moderate 5.4 (SD 2.0), and Poor 4.5 (SD $2.3), \mathrm{P}=0.009$; no significant trend was shown when the data were scored according to $\mathrm{LL} / \mathrm{HL}, \mathrm{P}=0.518$.

Nuclear pAKT staining (AS $\geq 1$ ) was detected in $56 / 101$ (55.4\%); 50/101 (49.5\%) showed HL staining. No significant correlations were detected with respect to PIK3CA mutation status, patient age, tumor stage, size or lymph node status ( $p>0.05)$. Nuclear AS data analysis showed an association with histological grade: Well 5.1 (SD 3.1), Moderate 3.3 (SD 3.0), and Poor 2.0 (SD 2.7), P = 0.014; no significant trend was shown when the data were scored according to $\mathrm{LL} / \mathrm{HL}, \mathrm{P}=0.085$. Representative staining is shown in Fig. 2.

\section{PIK3CA, PTEN, phospho-mTOR, phospho-p44/42 MAPK (ERK1/2)}

PIK3CA, PTEN, and p-mTOR, p-MAPK (ERK1/2) showed no significant relationships to mutation status or to any clinical parameter ( $\mathrm{P}>0.05)$. PIK3CA showed strong cytoplasmic staining (AS $\geq 4$ ) in 100/101 (99.0\%) specimens (Additional file 2: Figure S1). PTEN stained (mostly cytoplasmic, occasional nuclear) positive (AS $\geq 1)$ in $31 / 100(31.0 \%)$ specimens; $26 \%$ showed HL staining. mTOR stained (mostly cytoplasmic, occasional nuclear) positive $(\mathrm{AS} \geq 1)$ in $64 / 101(63.4 \%)$ specimens; $42 / 103(40.8 \%)$ showed HL staining. P-MAPK cytoplasmic staining was detected in 71/102 (69.6\%) and 59/102 (57.8\%) CSSCs (AS $\geq 1$ and AS $\geq 4$ respectively.) P-ERK1/2 nuclear staining was detected in 75/102 (73.5\%) and 64/102 (62.7\%) CSSCs (AS $\geq 1$ and AS $\geq 4$ respectively). Representative staining is shown in Fig. 2. 


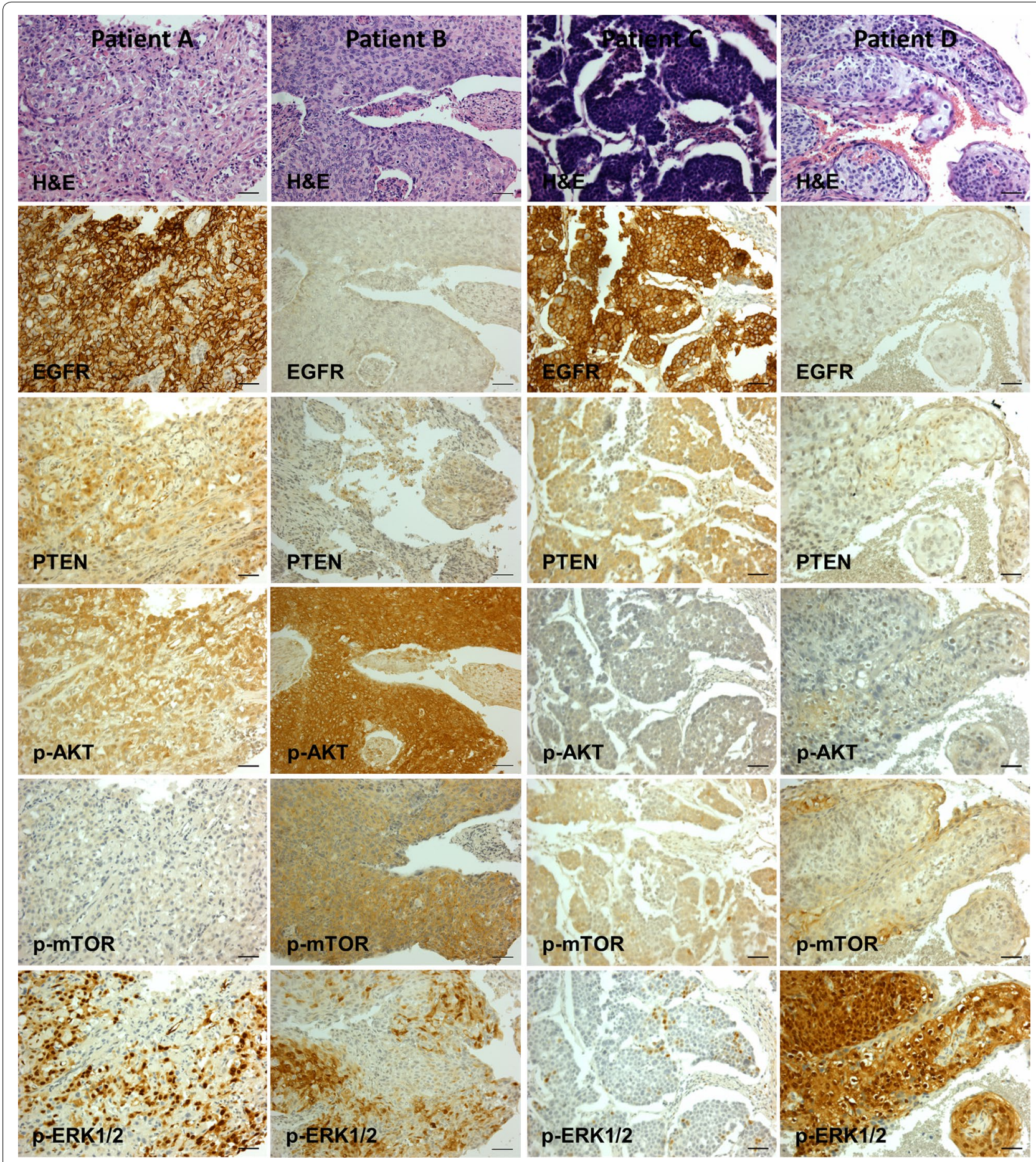

Fig. 2 Representative histochemical and immunohistochemical staining. Heterogeneous staining pattern combinations were detected among 105 cervical squamous cell carcinomas. Patients $A$ and $B$ : moderately differentiated Stage 1 tumors, wild type PIK3CA; patients C (poorly differentiated) and $D$ (moderately differentiated): Stage 1 tumor, mutated PIK3CA. Scale bar $50 \mu \mathrm{m}$. All images were originally photographed with a $\times 20$ objective lens using an Olympus BX50 light microscope (Center Valley, PA, USA) equipped with a Qlmaging Retiga 2000R digital camera (Surrey, BC, Canada) (PIK3CA staining is included in Additional File 2: Figure S1). 
Table 5 Summary of staining patterns that included statistically significant associations

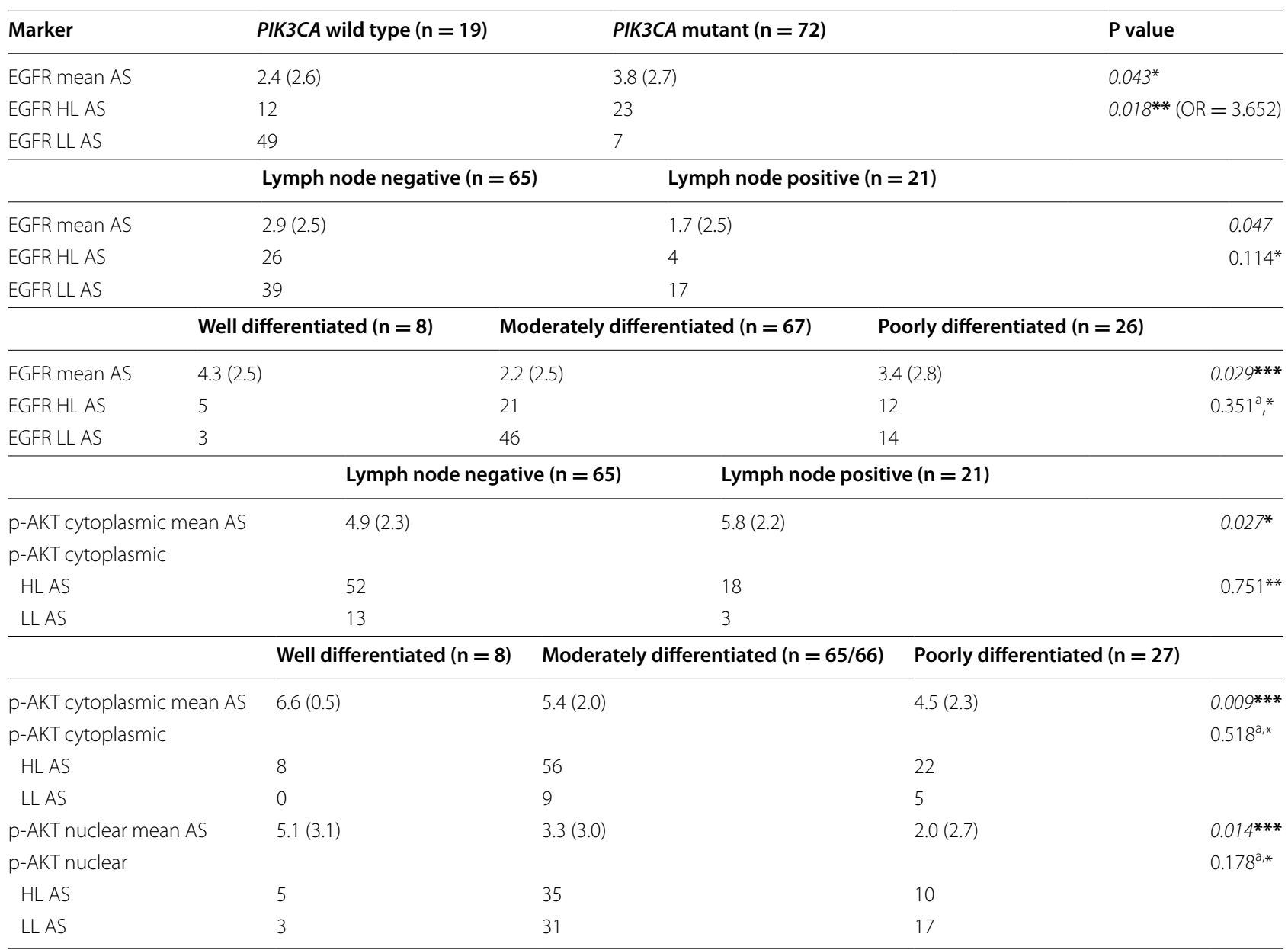

AS (Allred Score), High Level (HL) AS (AS $\geq 4$ ), Low Level (LL) AS (AS <4).

Italic values indicate statistically significant.

* Mann-Whitney test.

** Fisher's Exact test.

** Kruskall-Wallis test.

a Well and moderate categories combined.

\section{Inter-relationships of IHC markers}

EGFR over-expression may activate the PI3K/AKT/ mTOR pathway. No significant relationships were noted with respect to EGFR staining and PIK3CA, p-AKT or p-mTOR. Neither was PIK3CA staining related to p-AKT or p-mTOR, nor $\mathrm{p}-\mathrm{AKT}$ to $\mathrm{p}-\mathrm{mTOR}(\mathrm{P}<0.05)$. Furthermore, EGFR staining levels showed no correlation with pMAPK staining and there was also no significant association of PTEN expression with PIK3CA or pAKT.

\section{Discussion}

This study has investigated CSCC specimens for aberrations to key EGFR pathway elements. EGFR IHC staining patterns were examined in relation to $K R A S$ mutations and MAPK pathway expression, as well as in relation to PIK3CA mutations and PIK3CA, PTEN, pAKT and p-mTOR expression.

Low-level (LL) EGFR staining was present in $59.4 \%$ and high-level (HL) staining in $37.6 \%$ of the CSCC specimens. On the basis of mean Allred scores, EGFR staining was associated with well differentiated tumors and with lymph node negative patients. Previous EGFR IHC studies of cervical carcinomas show a wide range of expression levels $(6-100 \%)[16,18]$; these data may be a reflection of differences in antibody clone choices, IHC protocols, and/or in how staining was rated [16]. HPV genotype may also be important as HPV16 E5 or E6/E7 proteins can lead to EGFR overexpression [20,21]; our samples were all 
HPV16 positive, whereas other studies did not report or included a variety HPV types. There are also contradictory data about the relationship of EGFR expression to clinical markers, prognosis and therapeutic response: many studies show that EGFR overexpression is an independent predictor of poor response to radio/chemo-therapy, poor disease-free survival and poor overall survival; other studies show do not show these correlations [16]. Our data are supportive that EGFR staining could be used to identify CSCC patients eligible for anti-EGFR therapies.

The potential of anti-EGFR therapies for CSCC treatment is strengthened by the non-detection of KRAS mutations among our specimens. This finding is consistent with previous studies (Table 6) that have found KRAS mutations to be absent or uncommon (1.3-13.6\%) in CSCC; $K R A S$ mutations may occur more frequently in cervical adenocarcinomas (Table 6). KRAS mutations can chronically activate KRAS expression [9]. Via B-Raf, this in turn leads to continuously activated MAP kinase signaling as a consequence of phosphorylation in the cytoplasm of MEK1/2 and thence ERK1/2 and which in turn results in the activation of transcription factors promoting cell proliferation following migration into the cell nucleus [30]. In the absence of $K R A S$ mutations it might therefore be expected that over-expression of EGFR would correlate with the expression of phosphorylated ERK1/2; this type of invariable simple linear relationship was not detected
(Fig. 2). However, approximately 60\% (HL) to 75\% (LL) CSCCs stained negatively (cytoplasm or nucleus) suggesting the possibility that ERK1/2 staining might be used for identifying patients likely to benefit from anti-MAPK/ERK interventions. We are unaware of any other studies to date that have assessed pERK staining in cervical cancer.

PIK3CA mutations were detected in $20.0 \%$ of 95 CSCC specimens. As with IHC, mutation data estimates may be impacted by the experimental technique. Most studies of KRAS and PIK3CA mutations in cervical cancer have combined PCR amplification across mutation hotspot regions with Sanger sequencing (Table 6). The potential limitation of this approach is the detection sensitivity, which is of the order $\sim 10 \%$ [31]. The highest and lowest estimates of PIK3CA mutation frequencies in cervical cancers (5.7 and $37.5 \%$ ) have been obtained using Sequenom massARRAY technology [19, 32], which has a sensitivity threshold of $\sim 5 \%$ [33]. The detection sensitivity for methods employing massively parallel sequencing is of the order $\sim 1 \%$ [31], which is the same as the analytical sensitivity reported for the PNA-Clamp technique [34]. PIK3CA mutations may activate the PI3K-PTENAKT pathway; however, there are contradictory data as to whether or not PIK3CA mutations confer anti-EGFR therapy resistance [35-37].

The PIK3CA and KRAS mutation detection rates among the Indian CSCCs is comparable to what has been found

Table 6 KRAS and PIK3CA mutations in cervical cancer

\begin{tabular}{|c|c|c|c|c|}
\hline \multirow[t]{2}{*}{ References } & \multicolumn{2}{|c|}{ Squamous cell carcinoma } & \multicolumn{2}{|c|}{ Adenocarcinoma/other } \\
\hline & KRAS mutation & PIK3CA mutation & KRAS mutation & PIK3CA mutation \\
\hline Dokianakis et al. [50] ${ }^{a}$ & $0 / 10(0 \%)^{1}$ & - & $2 / 2(100 \%)^{1}$ & - \\
\hline Stenzel et al. [51] $]^{\mathrm{b}}$ & $3 / 22(13.6 \%)^{2}$ & - & $1 / 2(50.0 \%)^{2}$ & - \\
\hline Pappa et al. [52] $]^{a}$ & $2 / 28(7.1 \%)^{3}$ & - & $1 / 19(5.3 \%)^{3}$ & - \\
\hline Miyake et al. [53] ${ }^{\mathrm{C}}$ & - & $2 / 12(16.7 \%)^{i}$ & - & $1 / 9(11.1 \%)^{i}$ \\
\hline Cui et al. [38] ${ }^{d}$ & - & $5 / 84(6.0 \%)^{i i}$ & - & $10 / 100(10.0 \%)^{i i}$ \\
\hline lida et al. [18] ${ }^{c}$ & $0 / 32(0 \%)^{4}$ & - & $3 / 48(6.3 \%)^{4}$ & - \\
\hline Janku et al. [54] & - & i $2 / 8(25.0 \%)$ & - & $0 / 7(0 \%)^{i}$ \\
\hline Janku et al. [55] $]^{\mathrm{e}}$ & $1 / 10(10 \%)^{4}$ & $5 / 14(35.7 \%)^{i}$ & - & - \\
\hline Janku et al. [40] & - & $6 / 18(33.3 \%)^{i}$ & - & - \\
\hline Wright et al. [19] & $0 / 40(0 \%)^{5}$ & $15 / 40(37.5 \%)^{i i i}$ & $7 / 40(17.5 \%)^{5}$ & $10 / 40(25.0 \%)^{\mathrm{iii}}$ \\
\hline Mclntyre et al. [39] & - & $16 / 69(23.2 \%)^{i}$ & - & $3 / 13(23.1 \%)^{i}$ \\
\hline Ojesina et al. [48] & $1 / 79(1.3 \%)^{6}$ & $9 / 79(11.4 \%)^{i v}$ & $2 / 35(5.7 \%)^{6}$ & $8 / 35(22.9 \%)^{\mathrm{iv}}$ \\
\hline Rashmi et al. [32] & - & $6 / 120(5.0 \%)^{\mathrm{iii}}$ & - & $2 / 20(10.0 \%)^{\mathrm{iii}}$ \\
\hline Spaans et al. [47] ${ }^{\mathrm{h}}$ & $9 / 205^{\mathrm{ns}}(4.4 \%)^{5}$ & $50 / 205^{\mathrm{ns}}(24.4 \%)^{\mathrm{iii}}$ & Ns & Ns \\
\hline Tornesello et al. [56] & - & $3 / 55(5.5 \%)^{\vee}$ & - & - \\
\hline The present study ${ }^{j}$ & $0 / 91(0 \%)^{7}$ & $19 / 95(20.0 \%)^{v i}$ & - & - \\
\hline
\end{tabular}

Specimen country of origin: ${ }^{\mathrm{a}}$ Greece, ${ }^{\mathrm{b}}$ Poland, ${ }^{\mathrm{c}}$ Japan, ${ }^{\mathrm{d}}$ Sweden, ${ }^{\mathrm{e}}$ USA, ${ }^{\mathrm{f}}$ Canada, ${ }^{\mathrm{g}}$ Norway and Mexico, ${ }^{\mathrm{h}}$ The Netherlands, ${ }^{\mathrm{i}}$ Italy, and ${ }^{\mathrm{j}}$ India. $K R A S$ mutation methodology: ${ }^{1}$ codon 12 PCR/sequencing, ${ }^{2}$ codon 12 PCR-RFLP/SSCP, ${ }^{3}$ codons 12,13 and 16 PCR/sequencing, ${ }^{4}$ codons 12 and 13 PCR/sequencing, ${ }^{5}$ sequenom MALDI-TOFF massARRAY, ${ }^{6}$ exome/whole genome Illumina HiSeq $200,{ }^{7}$ codons 12 and 13 PNA-Clamp PCR. PIK3CA mutation methodology: ${ }^{i}$ exons 9 and 20 PCR/ sequencing, i exons 1, 9 and 20 PCR/sequencing, iii sequenom MALDI-TOF massARRAY, iv exome/whole genome Illumina HiSeq 200 , ${ }^{v}$ exon 9 nested PCR/sequencing,

vi exons 9 and 20 PNA-Clamp PCR. ${ }^{\text {ns }}$ not specified whether specimens were squamous cell carcinoma or adenocarcinoma. 
in MDC CSCCs (Table 6) suggesting that susceptibility to the accumulation of these mutations in CSCC aetiology is an intrinsic biologic property of the disease rather than related to ethnicity or culture. PIK3CA mutation detection in our study was associated with older, post-menopausal age. The possibility of a relationship to older age has previously been suggested on the basis of data combined from the detection of PIK3CA mutations in $5 / 84$ (6.0\%) CSCC and 10/100 (10.0\%) cervical adenocarcinomas in patients from Sweden [38] suggesting age may be a global determinant for PIK3CA mutation susceptibility in cervical cancer. These data indicate that PIK3CA mutation prevalence estimates may be 'biased' by the age of the sample population. Among our specimen set, PIK3CA mutations did not correlate with any available clinical parameters. In previous studies, PIK3CA mutations have been found associated with shorter survival [19], or with better survival or treatment response for patients treated with radical chemoradiotherapy or PI3K/AKT/mTOR inhibitors [39-41]. These findings together with the discovery of mutations in $20 \%$ of CSCCs are supportive for the use of PIK3CA mutation testing for prognostic stratification and as a potential target for therapeutic interventions. PIK3CA amplification has been reported in 14/20 (70.0\%) [42] CSCCs; however, by IHC PIK3CA staining was strong in 99.0\% CSCCs specimen in our study.

In relation to the EGFR/AKT/mTOR signaling pathway, PIK3CA mutations were associated with EGFR positive staining assessed either in in terms of AS $(\mathrm{P}=0.043)$ or by $L L$ vs. HL values ( $\mathrm{P}=0.018 ; \mathrm{OR}=3.652$ ). The finding of a significant association with either form of IHC rating, strengthens support for a relationship between EGFR expression and PIK3CA mutation acquisition. Possibly, continuous PI3K synthesis together with increased cell proliferation heightens susceptibility to a PIK3CA mutation event. These data are also suggestive that patients with EGFR overexpression and PIK3CA mutations might usefully be treated synergistically using drugs for both targets.

PTEN staining was detected in a minority of specimens and was not associated with any of the clinical parameters. Absence of expression of this tumor suppressor protein that dephosphorylates PIP3 preventing AKT activation is consistent with tumor promotion. Other studies have found low PTEN expression in CSCC but have also noted an association of expression with tumor size and lymph node involvement [43, 44]. Loss of heterozygosity at the PTEN locus has been shown up to $23 \%$ of Indian cervical cancers [45] and PTEN promoter methylation in 58\% of CSCC [46]. PTEN mutations in cervical cancer have been reported in 2.4-6.9\% of specimens [47, 48]. Accordingly, PTEN IHC biomarker data might also usefully be figured into a drug selection for CSCC.
Elevated EGFR, in the absence or presence of PTEN expression, did not correlate with elevated phospho-AKT and phospho-mTOR expression. Cytoplasmic pAKT expression was common and was associated with lymph node positive patients $(\mathrm{P}=0.022)$ and with well differentiated tumors when data was assessed in terms of AS values but no such association was found when the data was assessed by LL/HL cut-off points. Nuclear pAKT (detected in $\sim 50 \%$ of the tumors) also showed an association with well differentiated tumors by AS assessment only $(\mathrm{P}=0.046)$. $\mathrm{P}-\mathrm{mTOR}$ showed staining in $40.8 \%$ (HL) $-59.2 \%$ (LL) of the specimens and was unassociated with any study parameters.

In this study we focused on some of the factors that impact EGFR related pathways. Intercellular signaling pathways, such as the MAPK/ERK and PI3K/AKT/ mTOR, are circuitously inter-related and impacted directly or indirectly by multiple other factors, genetic and epigenetic. Consequently, the expression of downstream events in a pathway may be the outcome of other over-riding (aberrant) parallel pathway processes or meta-levels of regulation rather than being directly related to an upstream amplification or mutation event. For example, in the case of cervical disease, the expression of other RTKs such as ErbB-2, or enzymes such as cyclooxygenase-2 may impact pathway expression [57]. Additionally, CSCC results from chronic infection with high-risk HPV. The effects of the HPV16 E5, E6 and E7 oncogenes have been well characterized for their dysregulation of EGFR, p53 and pRB respectively. The HPV oncogenes can also impact multiple additional cellular pathway factors $[58,59]$. It is therefore unsurprising that CSCC shows manifold heterogeneity as reflected in EGFR pathway expression profiles.

In summary, the major findings of this study are threefold. Firstly, KRAS mutations were undetected in Indian CSSCs whereas PIK3CA mutations were ageassociated and relatively common being detected in $28.0 \%$ of specimens from women $\geq 50$ years vs. $11.1 \%$ of women $<50$ years. Secondly, across patients EGFR staining was common and was associated with PIK3CA mutation detection (by mean AS or by LL/HL scoring) and also showed associations with lymph node status and histological grade. pAKT IHC staining also showed association with lymph node status and histological grade. These relationships were partially dependent on how IHC staining patterns were scored reaffirming the requirement for standardized IHC protocols to allow accurate biomarker inter-study comparisons and clinical appraisal [49]. Thirdly, at the level of individual patients, qualitatively distinct marker panel staining profiles were observed patient to patient (Fig. 2). This finding may be of significance for precision/individualized/ 
personalized medicine. A variety of cellular pathways are candidates for pharmacologic interventions and for each pathway there may be multiple potential actionable targets. Our data suggest that in addition to molecular profiling, IHC data might be usefully incorporated into precision medicine algorithms; the expression level of putative pathway targets may be important for deciding among therapeutic options. For example, Patient A (Fig. 2) might be a candidate for EGFR or ERK targeted therapies, Patient B for AKT, mTOR or ERK, Patient C for EGFR or mTOR, and Patient D for ERK therapies. Clinical trials in particular might benefit from noting pre- and post-treatment expression levels of intervention pathways/targets and the relationship to therapeutic response.

\section{Conclusions}

This study shows that EGFR represents a promising target for CSCC treatments, especially given the absence of $K R A S$ mutations among the study samples. It is unclear whether PIK3CA mutations compromise EGFR therapies. However, pharmacologic agents used to treat CSCC patients with PIK3CA mutations have been shown to be efficacious [41]; these together with EGFR treatments might therefore be usefully combined. Downstream cellular pathway protein expression levels may not necessarily be predictable on the basis of amplification events or activating mutations. IHC or proteomic assay of these expression levels may be of importance for selecting among alternative therapeutic strategies for use in precision medicine protocols. Overall, the data for the Indian CSCCs is comparable to data published for CSCCs from more developed countries suggesting that EGFR pathway-related therapies could be of benefit to patients worldwide.

\section{Additional files}

Additional file 1: Table S1. KRAS and PIK3CA mutations detected.

Additional file 2: Figure S1. Representative histochemical and immunohistochemical staining. Heterogeneous staining pattern combinations were detected among 105 cervical squamous cell carcinomas. Patients $A$ and B: moderately differentiated Stage 1 tumors, wild type PIK3CA; patients C (poorly differentiated) and D (moderately differentiated): Stage 1 tumor, mutated PIK3CA. Scale bar: $50 \mu \mathrm{m}$. All images were originally photographed with a X20 objective lens using an Olympus BX50 light microscope Center Valley, PA) equipped with a QImaging Retiga 2000R digital camera (Surrey, BC).

\section{Abbreviations}

HPV: human papillomaviruses; CSCC: cervical squamous cell carcinoma; MDCs: more developed countries; LDCs: less developed countries; FFPE: formalinfixed, paraffin-embedded; PNA: peptide nucleic acid; TMA: tissue microarray; IHC: Immunohistochemistry; LN: Iymph node status; AS: Allred Score; LL: low-level; HL: high-level.

\section{Authors' contributions}

The study was designed and supervised by MFE. All laboratory assays were performed by SB. Data analyses, statistical tests and interpretations were by MFE and SB with supporting contributions from KC, TE and CP. Patient specimens, clinical data and histopathological review were provided by KM, SN and KC. The manuscript was written by MFE and SB with supporting contributions from $T E, C P, K C$ and $K M$. All authors read and approved the final manuscript.

\section{Author details}

${ }^{1}$ Department of Microbiology, Khon Kaen University, Khon Kaen 40002, Thailand. ${ }^{2}$ Department of Pathology, Sri Aurobindo Institute of Medical Sciences, Indore, Madhya Pradesh 453555, India. ${ }^{3}$ Department of Pathology and Laboratory Medicine, Pearlman School of Medicine, University of Pennsylvania, Philadelphia 19104-4283, USA. ${ }^{4}$ HPV \& EBV and Carcinogenesis Research Group, Khon Kaen University, Khon Kaen 40002, Thailand. ${ }^{5}$ Department of Pathology and Laboratory Medicine, University of Vermont, Burlington 05405, VT, USA.

${ }^{6}$ University of Vermont Cancer Center, Burlington, VT 05405, USA.

\section{Acknowledgements}

$\mathrm{PhD}$ candidate Ms. Sureewan Bumrungthai was supported for this research by the Thailand Royal Golden Jubilee Ph.D. Program (Grant No. PHD/0212/2550). The authors are grateful to Vanitha Rajendran, MSc, Zhihua Peng, PhD and Christine S-C Adamson, BS of the UVM Experimental Pathology Laboratory and Kendall E Black, BS, of Pulmonary Medicine for technical advice and assistance. We also wish to thank the UVM Microscopy Imaging Center for access to microscopy and imaging facilities.

\section{Compliance with ethical guidelines}

\section{Competing interests}

The authors declare that they have no competing interests.

Received: 10 February 2015 Accepted: 17 July 2015

Published online: 25 July 2015

\section{References}

1. Ferlay J, Soerjomataram I, Ervik M, Dikshit R, Eser S, Mathers C, et al (2013) GLOBOCAN 2012 v1.0, Cancer Incidence and Mortality Worldwide: IARC CancerBase No. 11 [Internet]. International Agency for Research on Cancer, Lyon. http://www.globocan.iarc.fr/Pages/fact_sheets_cancer.aspx (cervix uteri). Accessed 15 July 2015

2. Bhaumik S (2013) India has the world's highest number of cervical cancer death. BMJ 346:f3108. http://www.bmj.com/content/346/bmj.f3108. Accessed 15 July 2015

3. National Cancer Institute at the National Institute of Health (2013) Cervical cancer screening $\left(\mathrm{PDQ}^{\circledR}\right)$. http://www.cancer.gov/cancertopics/pdq/ screening/cervical/HealthProfessional/page2.2015. Accessed 15 July 2015

4. Chhabra S, Bhavani M, Mahajan N, Bawaskar R (2010) Cervical cancer in Indian rural women: trends over two decades. J Obstet Gynaecol 30:725-728

5. Senapathy JG, Umadevi P, Kannika PS (2011) The present scenario of cervical cancer control and HPV epidemiology in India: an outline. Asian Pac J Cancer Prev 12:1107-1115

6. National Cancer Institute Surveillance, Epidemiology, and End Results Program (2011) SEER stat fact sheets: cervix uteri cancer. http://www.seer. cancer.gov/statfacts/html/cervix.html. Accessed 15 July 2015

7. Yewale C, Baradia D, Vhora I, Patil S, Misra A (2013) Epidermal growth factor receptor targeting in cancer: a review of trends and strategies. Biomaterials 34:8690-8707

8. Seshacharyulu P, Ponnusamy MP, Haridas D, Jain M, Ganti AK, Batra SK (2012) Targeting the EGFR signaling pathway in cancer therapy. Expert Opin Ther Targets 16:15-31

9. Berg M, Soreide K (2012) EGFR and downstream genetic alterations in KRAS/BRAF and PI3K/AKT pathways in colorectal cancer: implications for targeted therapy. Discov Med 14:207-214

10. Meriggi F, Di Biasi B, Abeni C, Zaniboni A (2009) Anti-EGFR therapy in colorectal cancer: how to choose the right patient. Curr Drug Targets 10:1033-1040 
11. Roberts PJ, Der CJ (2007) Targeting the Raf-MEK-ERK mitogen-activated protein kinase cascade for the treatment of cancer. Oncogene 26:3291-3310

12. Markman B, Dienstmann R, Tabernero J (2010) Targeting the PI3K/Akt/ mTOR pathway-beyond rapalogs. Oncotarget 1:530-543

13. Polivka J Jr, Janku F (2014) Molecular targets for cancer therapy in the PI3K/AKT/mTOR pathway. Pharmacol Ther 142:164-175

14. Chong CR, Janne PA (2013) The quest to overcome resistance to EGFRtargeted therapies in cancer. Nat Med 19:1389-1400

15. Janku F, Hong DS, Fu S, Piha-Paul SA, Naing A, Falchook GS et al (2014) Assessing PIK3CA and PTEN in early-phase trials with PI3K/AKT/mTOR inhibitors. Cell Rep 6:377-387

16. Soonthornthum T, Arias-Pulido H, Joste N, Lomo L, Muller C, Rutledge T et al (2011) Epidermal growth factor receptor as a biomarker for cervical cancer. Ann Oncol 22:2166-2178

17. Arias-Pulido H, Joste N, Chavez A, Muller CY, Dai D, Smith HO et al (2008) Absence of epidermal growth factor receptor mutations in cervical cancer. Int J Gynecol Cancer 18:749-754

18. Iida K, Nakayama K, Rahman MT, Rahman M, Ishikawa M, Katagiri A et al (2011) EGFR gene amplification is related to adverse clinical outcomes in cervical squamous cell carcinoma, making the EGFR pathway a novel therapeutic target. Br J Cancer 105:420-427

19. Wright AA, Howitt BE, Myers AP, Dahlberg SE, Palescandolo E, Van Hummelen P et al (2013) Oncogenic mutations in cervical cancer: genomic differences between adenocarcinomas and squamous cell carcinomas of the cervix. Cancer 119:3776-3783

20. Tsai TC, Chen SL (2003) The biochemical and biological functions of human papillomavirus type 16 E5 protein. Arch Virol 148:1445-1453

21. Akerman GS, Tolleson WH, Brown KL, Zyzak LL, Mourateva E, Engin TS et al (2001) Human papillomavirus type 16 E6 and E7 cooperate to increase epidermal growth factor receptor (EGFR) mRNA levels, overcoming mechanisms by which excessive EGFR signaling shortens the life span of normal human keratinocytes. Cancer Res 61:3837-3843

22. Spangle JM, Munger K (2013) The HPV16 E6 oncoprotein causes prolonged receptor protein tyrosine kinase signaling and enhances internalization of phosphorylated receptor species. PLoS Pathog 9:e1003237

23. Morris EJ, Jha S, Restaino CR, Dayananth P, Zhu H, Cooper A et al (2013) Discovery of a novel ERK inhibitor with activity in models of acquired resistance to BRAF and MEK inhibitors. Cancer Discov 3:742-750

24. Ganesan P, Janku F, Naing A, Hong DS, Tsimberidou AM, Falchook GS et al (2013) Target-based therapeutic matching in early-phase clinical trials in patients with advanced colorectal cancer and PIK3CA mutations. Mol Cancer Ther 12:2857-2863

25. Wang L, Hu H, Pan Y, Wang R, Li Y, Shen L et al (2014) PIK3CA mutations frequently coexist with EGFR/KRAS mutations in non-small cell lung cancer and suggest poor prognosis in EGFR/KRAS wildtype subgroup. PLoS One 9:e88291

26. Mester J, Eng C (2013) When overgrowth bumps into cancer: the PTENopathies. Am J Med Genet C 163C:114-121

27. Munjal K, Adamson CSC, Rajendran V, Nandedkar S, Cooper K, Evans MF (2014) Human papillomavirus type distribution in invasive cervical cancers From Madhya Pradesh: Implications for vaccination programs in Central India. Int J Gynecol Pathol 33:531-536

28. Choudhury KR, Yagle KJ, Swanson PE, Krohn KA, Rajendran JG (2010) A robust automated measure of average antibody staining in immunohistochemistry images. J Histochem Cytochem 58:95-107

29. Conant JL, Peng ZH, Evans MF, Naud S, Cooper K (2011) Sarcomatoid renal cell carcinoma is an example of epithelial-mesenchymal transition. J Clin Pathol 64:1088-1092

30. Zassadowski F, Rochette-Egly C, Chomienne C, Cassinat B (2012) Regulation of the transcriptional activity of nuclear receptors by the MEK/ERK1/2 pathway. Cell Signal 24:2369-2377

31. Herreros-Villanueva M, Chen CC, Yuan SS, Liu TC, Er TK (2014) KRAS mutations: analytical considerations. Clin Chim Acta 431:211-220

32. Rashmi R, DeSelm C, Helms C, Bowcock A, Rogers BE, Rader J et al (2014) AKT inhibitors promote cell death in cervical cancer through disruption of mTOR signaling and glucose uptake. PLoS One 9:e92948

33. Pearce M, Ehrich M (2010) Somatic mutation analysis in tumor samples using the SequenomMassARRAY ${ }^{\circledR}$ system. Nature Methods Application Notes. http://www.nature.com/app_notes/nmeth/2010/101304/full/ an7545.html\#References. Published online 17 April 2010. Accessed 15 July 2015
34. Kobunai T, Watanabe T, Yamamoto Y, Eshima K (2010) The frequency of KRAS mutation detection in human colon carcinoma is influenced by the sensitivity of assay methodology: a comparison between direct sequencing and real-time PCR. Biochem Bioph Res Co 395:158-162

35. DeRoock W, Claes B, Bernasconi D, De Schutter J, Biesmans B, Fountzilas $G$ et al (2010) Effects of KRAS, BRAF, NRAS, and PIK3CA mutations of the efficacy of cetuximab plus chemotherapy in chemotherapy-refractory metastatic colorectal cancer: a retrospective consortium analysis. Lancet Oncol 11:753-762

36. Sartore-Bianchi A, Martini M, Molinari F, Veronese S, Nichelatti M, Artale S et al (2009) PIK3CA mutations in colorectal cancer are associated with clinical resistance to EGFR-targeted monoclonal antibodies. Cancer Res 69:1851-1857

37. Prenan H, DeSchutter J, Jacobs B, De Roock W, Biesmans B, Claes B, Lambrechts D et al (2009) PIK3CA mutations are not a major determinant of resistance to the epidermal growth factor inhibitor cetuximab in metastatic colorectal cancer. Clin Cancer Res 15:3184-3188

38. Cui B, Zheng B, Zhang X, Stendahl U, Andersson S, Wallin KL (2009) Mutation of PIK3CA: possible risk factor for cervical carcinogenesis in older women. Int J Oncol 34:409-416

39. McIntyre JB, Wu JS, Craighead PS, Phan T, Kobel M, Lees-Miller SP et al (2013) PIK3CA mutational status and overall survival in patients with cervical cancer treated with radical chemoradiotherapy. Gynecol Oncol 128:409-414

40. Janku F, Wheler JJ, Naing A, Stepanek VM, Falchook GS, Fu S et al (2012) PIK3CA mutations in advanced cancers: characteristics and outcomes. Oncotarget 3:1566-1575

41. Hou MM, Liu X, Wheler J, Naing A, Hong D, Coleman RL et al (2014) Targeted PI3K/AKT/mTOR therapy for metastatic carcinomas of the cervix: A phase I clinical experience. Oncotarget 30:11168-11179

42. Bertelsen Bl, Steine SJ, Sandvei R, Molven A, Laerum OD (2006) Molecular analysis of the PI3K-AKT pathway in uterine cervical neoplasia: frequent PIK3CA amplification and AKT phosphorylation. Int J Cancer 118:1877-1883

43. Lu D, Qian J, Yin X, Xiao Q, Wang C, Zeng Y (2012) Expression of PTEN and survivin in cervical cancer: promising biological markers for early diagnosis and prognostic evaluation. Brit J Biomed Sci 69:143-146

44. Eijsink JJ, Noordhuis MG, ten Hoor KA, Kok M, Hollema H, de Bock GH et al (2010) The epidermal growth factor receptor pathway in relation to pelvic lymph node metastasis and survival in early-stage cervical cancer. Hum Pathol 41:1735-1741

45. Rizvi MM, Alam MS, Mehdi SJ, Ali A, Batra S (2012) Allelic loss of 10q23.3, the PTEN gene locus in cervical carcinoma from Northern Indian population. Pathol Oncol Res 18:309-313

46. Cheung TH, Lo KW, Yim SF, Chan LK, Heung MS, Chan CS et al (2004) Epigenetic and genetic alternation of PTEN in cervical neoplasm. Gynecol Oncol 93:621-627

47. Spaans VM, Trietsch MD, Crobach S, Stelloo E, Kremer D, Osse EM et al (2014) Designing a high-throughput somatic mutation profiling panel specifically for gynaecological cancers. PLoS One 9:e93451

48. Ojesina Al, Lichtenstein L, Freeman SS, Pedamallu CS, Imaz-Rosshandler I, Pugh TJ et al (2014) Landscape of genomic alterations in cervical carcinomas. Nature 506:371-375

49. Goldstein NS, Hewitt SM, Taylor CR, Yaziji H, Hicks DG (2007) Recommendations for improved standardization of immunohistochemistry. Appl Immunohisto M M 15:124-133

50. Dokianakis DN, Sourvinos G, Sakkas S, Athanasiadou E, Spandidos DA (1998) Detection of HPV and ras gene mutations in cervical smears from female genital lesions. Oncol Rep 5:1195-1198

51. Stenzel A, Semczuk A, Rozynskal K, Jakowicki J, Wojcierowski J (2001) "Low-risk" and "high-risk" HPV-infection and K-ras gene point mutations in human cervical cancer: a study of 31 cases. Pathol Res Pract 197:597-603

52. Pappa Kl, Choleza M, Markaki S, Giannikaki E, Kyroudi A, Vlachos G et al (2006) Consistent absence of BRAF mutations in cervical and endometrial cancer despite KRAS mutation status. Gynecol Oncol 100:596-600

53. Miyake T, Yoshino K, Enomoto T, Takata T, Ugaki H, Kim A et al (2008) PIK3CA gene mutations and amplifications in uterine cancers, identified by methods that avoid confounding by PIK3CA pseudogene sequences Cancer Lett 261:120-126 
54. Janku F, Tsimberidou AM, Garrido-Laguna I, Wang X, Luthra R, Hong DS et al (2011) PIK3CA mutations in patients with advanced cancers treated with PI3K/AKT/mTOR axis inhibitors. Mol Cancer Ther 10:558-565

55. Janku F, Lee JJ, Tsimberidou AM, Hong DS, Naing A, Falchook GS et al (2011) PIK3CA mutations frequently coexist with RAS and BRAF mutations in patients with advanced cancers. PLoS One 6:e22769.08

56. Tornesello M, Annunziata C, Buonaguro L, Losito S, Greggi S, Buonaguro FM (2014) TP53 and PIK3CA gene mutations in adenocarcinoma, squamous cell carcinoma and high-grade intraepithelial neoplasia of the cervix. J Transl Med 12:255
57. Fukazawa EM, Baiocchi G, Soares FA, Kumagai LY, Faloppa CC, BadiglianFilho L et al (2014) Cox-2, EGFR, and ERBB-2 expression in cervical intraepithelial neoplasia and cervical cancer using an automated imaging system. Int J Gynecol Pathol 33:225-234

58. Moody CA, Laimins LA (2010) Human papillomavirus oncoproteins: pathways to transformation. Nat Rev Cancer 10:550-560

59. de Freitas AC, Coimbra EC, Leitão Mda C (2014) Molecular targets of HPV oncoproteins: potential biomarkers for cervical carcinogenesis. Biochim Biophys Acta 1845:91-103
Submit your next manuscript to BioMed Central and take full advantage of:

- Convenient online submission

- Thorough peer review

- No space constraints or color figure charges

- Immediate publication on acceptance

- Inclusion in PubMed, CAS, Scopus and Google Scholar

- Research which is freely available for redistribution

Submit your manuscript at www.biomedcentral.com/submit
() BioMed Central 